\title{
Potential for Integrating Entry Guidance into the Multi-Disciplinary Entry Vehicle Optimization Environment
}

\author{
Sarah D’Souza*, David J. Kinney ${ }^{\dagger}$, Joseph A. Garcia ${ }^{\dagger}$ \\ NASA Ames Research Center, Moffett Field, California, 94035 \\ Nesrin Sarigul-Klijn ${ }^{\ddagger}$ \\ Space Engineering Research and Graduate Program (SpaceED) \\ Mechanical and Aerospace Engineering Department, University of California Davis \\ Davis, CA 95616-5294
}

\begin{abstract}
The state-of-the-art in vehicle design decouples flight feasible trajectory generation from the optimization process of an entry spacecraft shape. The disadvantage to this decoupled process is seen when a particular aeroshell does not meet in-flight requirements when integrated into Guidance, Navigation, and Control simulations. It is postulated that the integration of a guidance algorithm into the design process will provide a real-time, rapid trajectory generation technique to enhance the robustness of vehicle design solutions. The potential benefit of this integration is a reduction in design cycles (possible cost savings) and increased accuracy in the aerothermal environment (possible mass savings). This work examines two aspects: 1) the performance of a reference tracking guidance algorithm for five different geometries with the same reference trajectory and 2) the potential of mass savings from improved aerothermal predictions. An Apollo Derived Guidance (ADG) algorithm is used in this study. The baseline geometry and five test case geometries were flown using the same baseline trajectory. The guided trajectory results are compared to separate trajectories determined in a vehicle optimization study conducted for NASA's Mars Entry, Descent, and Landing System Analysis. This study revealed several aspects regarding the potential gains and required developments for integrating a guidance algorithm into the vehicle optimization environment. First, the generation of flight feasible trajectories is only as good as the robustness of the guidance algorithm. The set of dispersed geometries modelled aerodynamic dispersions that ranged from $+/-1 \%$ to $+/-17 \%$ and a single extreme case was modelled where the aerodynamics were approximately $80 \%$ less than the baseline geometry. The ADG, as expected, was able to guide the vehicle into the aeroshell separation box at the target location for dispersions up to $17 \%$, but failed for the $80 \%$ dispersion cases. Finally, the results revealed that including flight feasible trajectories for a set of dispersed geometries has the potential to save mass up to $430 \mathrm{~kg}$.
\end{abstract}

\section{Introduction}

Advances in optimization algorithms and computational capabilities have lead to the development of Multi-Disciplinary Design, Analysis, and Optimization (MDAO) environments for entry spacecraft design. MDAO environments integrate models from multiple fields of interest to generate candidate vehicle concepts that meet given mission criteria. The objective functions in entry vehicle optimization can be a function of trajectory dependent variables, for example, minimizing peak heat rate where heat rate is a

\footnotetext{
*Graduate NASA Pathways Intern, NASA Ames Research Center, Moffett Field, and AIAA Student Member.

${ }^{\dagger}$ Corresponding Authors. Aerospace Engineer, MS258-1, Systems Analysis and Design Branch/AUS, and Senior Member AIAA.

$\ddagger$ Corresponding Author. Professor and Director of SpaceED, UCDavis, MAE Department, Davis, CA 95616-5294, AIAA Associate Fellow, nsarigulklijn@ucdavis.edu 530-752-06822
} 
function of the altitude-velocity condition. The MDAO environment may include parametric vehicle shapes, trajectory optimization, structures models, and thermal protection system (TPS) sizing models. The aeordynamic/aerothermodynamic databases for the 'optimal' candidate geometry are provided to Guidance, Navigation, and Control (GNC) engineers who develop relevant guidance and control schemes to achieve trajectory specific mission criteria. GNC engineers complete dispersion analyses to test the perforrnance of GNC algorithms in meeting targeting requirements. The dispersions include, but are not limited to, uncertainties in navigational position, small changes in entry interface conditions, and uncertainty in aerodynamic quantities. The dispersion analysis generates a large database of heating and loading profiles corresponding to each dispersed trajectory. This database of trajectories is passed back to the MDAO tool to improve the candidate vehicle shape and TPS configuration.

The trajectory model in most MDAO environments does not necessarily determine a new trajectory when the geometry changes. In addition, the trajectory model does not always guarantee that a flight feasible trajectory is used to determine trajectory dependent parameters, such as heat rate. Herein, a flight feasible trajectory is defined as a profile that obeys the control limits and meets the targeting requirements. The use of trajectories that are not updated or not flight feasible may lead to an over-designed TPS configuration and may not reveal critical design errors that are eventually found after testing a physical prototype.

This research investigates the performance of an Apollo-Derived Guidance ${ }^{1}$ algorithm in the presence of dispersed aerodynamics due entirely to changing the entry vehicle geometry, which is what occurs in a shape optimization study. The use of a guidance algorithm as a trajectory model provides the potential to rapidly update the trajectory for a new geometry and ensure that a flight feasible trajectory is generated.

\section{I.A. Background}

There are multiple shape optimization studies that have been completed, where some may or may not include trajectory models or trajectory dependent objective functions. In Theisinger et al. ${ }^{2}$ a set of analytical geometric functions were implemented into an optimizer to study multiple shapes that minimize the ballistic coefficient while maintaining a specific L/D. However, no trajectory dependent variables were considered in the shape optimization, e.g. heat rate and/or heat load. There are some shape optimization studies that use aerothermodynamic analyses to optimize shapes. In Johnson et al. ${ }^{3}$, the shape of hypersonic blunt-body heat shields were optimized by minimizing total heat rate at the stagnation point. The vehicle speed and altitude are based on a single representative trajectory, thus flight feasible trajectories were not determined as the shape changed in the optimization. Another MDAO tool, called HAVOC ${ }^{4}$, was used to propose a conceptual design for a TPS material called Ultra High Temperature Ceramics (UHTC). HAVOC contained a 3DOF trajectory model consisting of two methods to compute the trajectories. The first is an energy state approximation method coupled with a Mach number vs. altitude scheduling. The second method solves the equations of motion with 1st order integration subject to a user specified set of path constraints. This tool provided engineers with the ability to complete a number of design cycles such that an optimum design was determined. However, the trajectory analysis lacked flight-feasible bank profiles and thus does not account for scenarios which may adversely affect the vehicle shape and TPS design. In Garcia et al. ${ }^{5}$ an MDAO tool, called Co-optimization Bluntbody Re-Entry Analysis (COBRA), was used to determine optimal aeroshell shapes for Mars atmospheric entry. COBRA includes parametric geometry models, aerodynamic models, aerothermodynamics models, trajectory optimization, TPS analysis tools, structures analysis, and mass estimation models. The objective functions were to minimize peak heat rate and maximize CDA to minimize the ballistic coefficient for a mid-L/D shape. While this shape optimization includes almost all disciplines including trajectory optimization, the trajectory analysis does not necessarily determine flight feasible trajectories. In Steinfeldt et al. ${ }^{6}$, a vehicle architecture landing assessment is completed with the integration of, among other models, a trajectory model. It is specifically stated in this paper that a close-loop guidance algorithm was not be included in the analysis because it would constrain the predicted performance of the system architecture. However, it can be argued that modelling closed loop guidance will provide the engineer with a realistic study of the performance that is needed to achieve the mission objectives. In Loomis et al. ${ }^{7}$ and Saunders et al. ${ }^{8}$, a TPS sizing tool was developed that integrates aerodynamics, aerothermodynamics, and trajectory analysis. In Loomis ${ }^{7}$ the size of a TPS aeroshell for the Mars Smart Lander was baselined. The authors use trajectory optimization and TPS sizing to achieve this. A constraint on the distance to the target landing site is included, with the potential to include this value in the objective function. However, the study conducted focuses on maximizing cross

range instead of achieving a precision landing. In addition, there does not appear to be any constraints 
on control rate or control acceleration and does not indicate how long this simulation takes to converge to a solution. Another tool for optimizing vehicle shapes is found in two papers authored by Kinney et al. ${ }^{9,10}$. The Configuration Based Aerodynamics (CBAERO) tool was used to optimize the HL-20 vehicle shape by maximizing L/D while holding volume constant and constraining the pitching moment to zero. In this study, no trajectory dependent parameters were used as constraints or objective functions. In Johnson et al. ${ }^{11}$, a population-based differential evolutionary algorithm was used to determine the optimal vehicle design for the multi-objective function to minimize heat load and maximize cross range. The class of vehicles under consideration ranged from an L/D of 0.3 to 1 . The authors selected entry conditions similar to the Orion spacecraft and used POST to model the trajectory. The bank profile was assumed to have a step function behavior. An initial guess of the bank profile was input and then bank angle adjustments were made through trial and error, until a trajectory was generated to meet the constraints. The trajectories were not necessarily flight feasible trajectories since targeting and bank limits (rate/acceleration) were not included. Clearly many MDAO studies lack the verification of flight feasible trajectories or any update once the trajectory changes. This gap in trajectory modelling for entry vehicle shape optimization is the motivation for studying guidance performance in the presence of this type of aerodynamic dispersion.

\section{Method}

As a pre-cursor to full integration, this study aims to determine the potential advantages to integrating a guidance algorithm into an entry vehicle MDAO environment. It is not within the scope of this study to develop a guidance algorithm or complete a full entry vehicle analysis. The general test case for this study is a Mars direct entry of a rigid mid-L/D aeroshell to land a 40 metric ton payload.

\section{II.A. Guidance and Trajectory Modeling}

In order to understand the limitations and/or potential of integrating current guidance algorithms into an MDAO environment, the Apollo Derived Guidance (ADG) algorithm was selected for this study. The ADG was used for Apollo's atmospheric entry and is widely used by the entry spacecraft GNC community. The ADG is a reference tracking algorithm with a terminal point controller derived using the calculus of variations for linear perturbations. The reference trajectory consists of drag acceleration, altitude rate, range to go, vertical L/D, and controller gains as a function of relative velocity. The application of the ADG in this work will provide an initial understanding of guidance performance in the presence of aerodynamic dispersions that are due entirely to a change in geometry. Although trajectory design is outside the scope of this study,
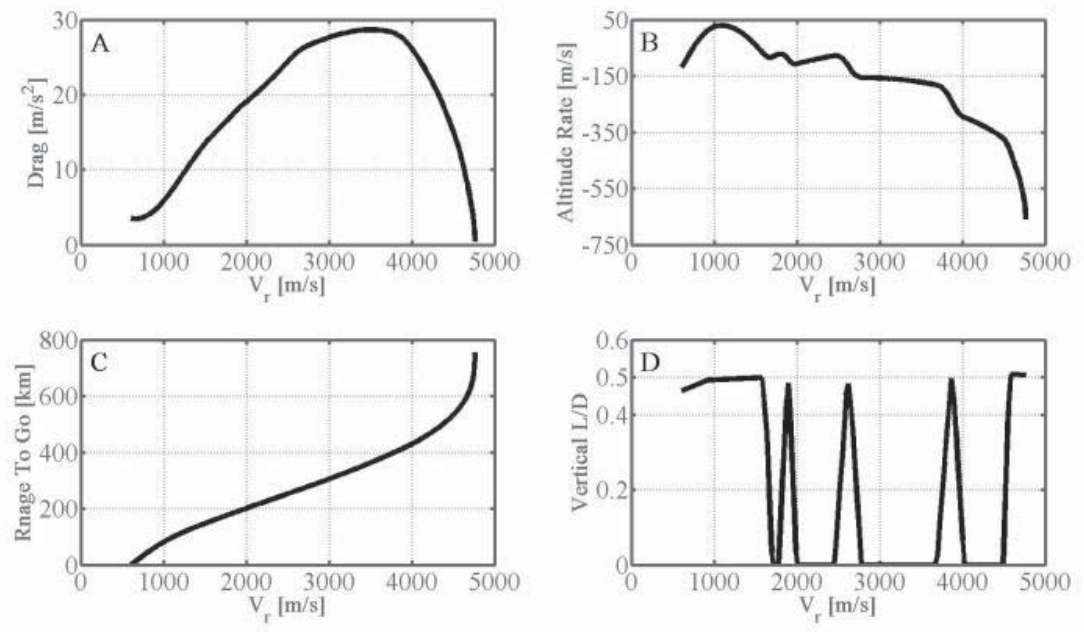

Figure 1. A) Reference drag acceleration, B) altitude rate, C) range to go, and D) vertical L/D versus relative velocity.

selecting an appropriate reference trajectory for implementation into the ADG is critical. The reference trajectory must ensure that the vehicle reaches its target and that the control profile does not violate control system constraints. In addition, the trajectory must ensure that aerodynamic loading and heating constraints 
are not violated. A nominal trajectory for a baseline mid-L/D vehicle shape was designed by NASA Langley Research Center that meets these criteria. This trajectory and corresponding geometry can be found in NASA's comprehensive Mars Entry, Descent, and Landing Systems Analysis. ${ }^{12}$ This baseline geometry and trajectory was implemented into the ADG for this work. This baseline is well suited for this study since the results will be compared with an entry vehicle MDAO study that used the same baseline case. The reference trajectory variables for the ADG are plotted in Figure 1.

The guidance algorithm is run in the Simulation of Rocket Trajectories (SORT) program. ${ }^{17}$ MarsGRAM 2005 is used to model the Mars atmosphere, the gravity model accounts for J2, J3, J4 -harmonics, and the aerodynamic and aerothermodynamic databases are acquired from CBAERO. ${ }^{10}$ The databases are a function of Mach number, dynamic pressure, and angle of attack, where angle of attack is fixed for each vehicle. The termination criteria (Table 1) correspond to separation of the aeroshell for the descent phase. In addition, the aerodynamic loading and heating constraints are monitored for each trajectory according to specified limits (Table 1).

\begin{tabular}{|l||c||c||c|}
\hline Separation & $h[\mathrm{~km}]$ & $q\left[\mathrm{~N} / \mathrm{m}^{2}\right]$ & Mach\# \\
\hline Lower & 6.4 & 1077.7 & 2.4 \\
\hline Upper & 8.9 & 1598.3 & 3.3 \\
\hline Maximum & $q\left[\mathrm{~N} / \mathrm{m}^{2}\right]$ & Loading $\left[\mathrm{g}^{\prime} \mathrm{s}\right]$ & $\mathrm{q}^{\prime}\left[\mathrm{W} / \mathrm{cm}^{2}\right]$ \\
\hline & 15000 & 4.0 & 1000.0 \\
\hline
\end{tabular}

Table 1. Trajectory parameters and termination criteria. ${ }^{5,12}$

\section{II.B. Vehicle Shapes and Aerodynamic Dispersions}

The total entry mass for the baseline geometry is 109.59 metric tons corresponding to a ballistic coefficient of $401.7 \mathrm{~kg} / \mathrm{m}^{2}$. Figure 2 illustrates the geometry with speed breaks and flaps, however, these control surface deflections were set to zero to generate the baseline aerodyamic and aerothermodynamic databases. This geometry will be referred to as the baseline or 'sled' throughout this paper. From this point forward, dispersed
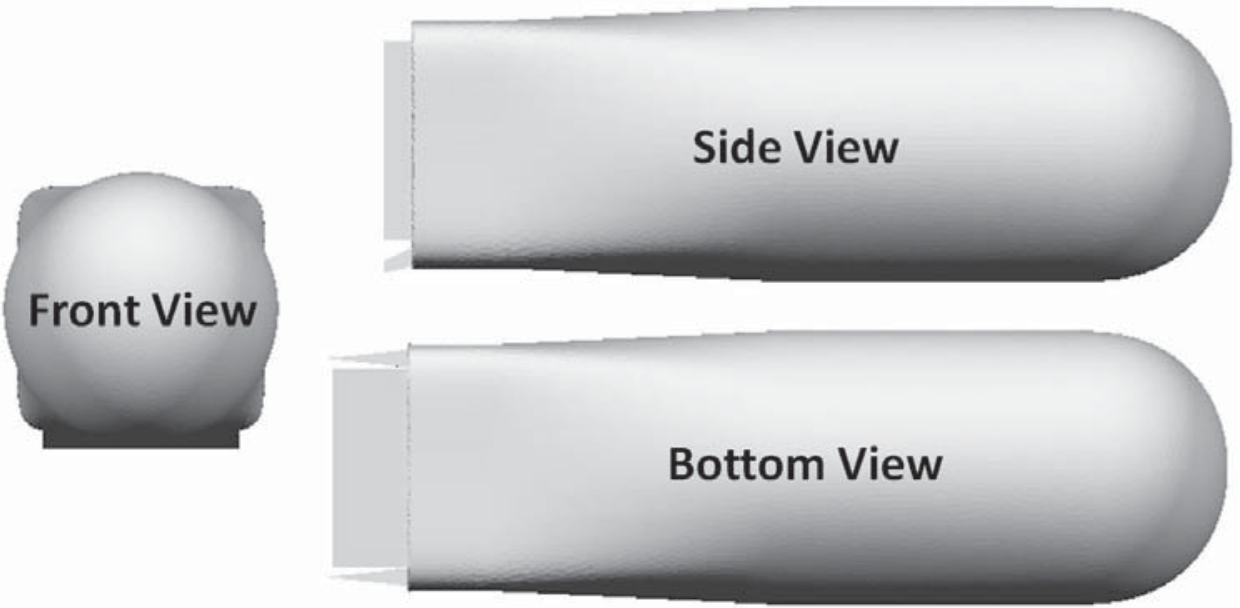

Figure 2. Baseline Sled Geometry

geometries will refer to 5 varied geometries generated from a comprehensive MDAO study of rigid, mid-L/D aeroshells. The target L/D for all geometries is 0.5 , where the change in geometry results in a change in the lift and drag coefficients but not the resulting ratio. In addition, the reference area and reference length for four of the five dispersed geometries is 78.54 square meters and 10 meters, respectively. In Garcia ${ }^{5}$, the aeroshell design space was explored by wrapping a Multi-Objective Genetic Optimization around an integrated set of relevant models. The Co-Optimization of Blunt-body Re-entry Analysis (COBRA) technique parametrically 
changes the aeroshell shape and uses a single nominal trajectory to define the aerodynamics loading and heating characteristics. Essentially, a single peak heating 'altitude-velocity' condition corresponding to a single reference geometry, is used to calculate the heat rate for each candidate geometry. The point of departure (POD) geometry, which is a spherical nose attached to a cylinder (Figure 3A), is the starting point for the parametric changes. The objective functions were to maximize $C_{D} A$ and minimize peak heating. The constraints included the launch shroud envelope and static stability for pitch and yaw. The resulting pareto front from the genetic optimization is a scatter plot of the total peak heat rate and $C_{D} A$ for each geometry in the design space. In the COBRA study, three candidate vehicles were selected such that the trade off between heat rate and $C_{D} A$ were considered. In Garcia ${ }^{5}$, these three candidate geometries were then run through the COBRA process again, but with some modifications. First, a pre-processed trajectory is optimized to match the altitude-velocity profile of the baseline trajectory. This trajectory does constrain the aerodynamic loading and heating, however, there are no constraints on the controls. Also, short of targeting a particular termination longitude and latitude for the direct entry portion of flight, the trajectory was constrained to ensure that the downrange was larger than $1220 \mathrm{~km}$. The trajectory analysis for the COBRA work included aerocapture and direct entry. The study in this paper focuses on the direct entry for the dispersed geometries. Second, a mass closure model iterates on the total entry mass until the subsystem masses (structure, TPS, etc.) converges. At this point a comparison of the aerodynamic loading, heating characteristics and total mass is completed to determine the "optimal" vehicle design. Four of the five dispersed geometries used in this study include the POD and the three candidate geometries selected from the COBRA study. It should be noted that the aerodynamic dispersion is a deviation from the $C_{L}$ and

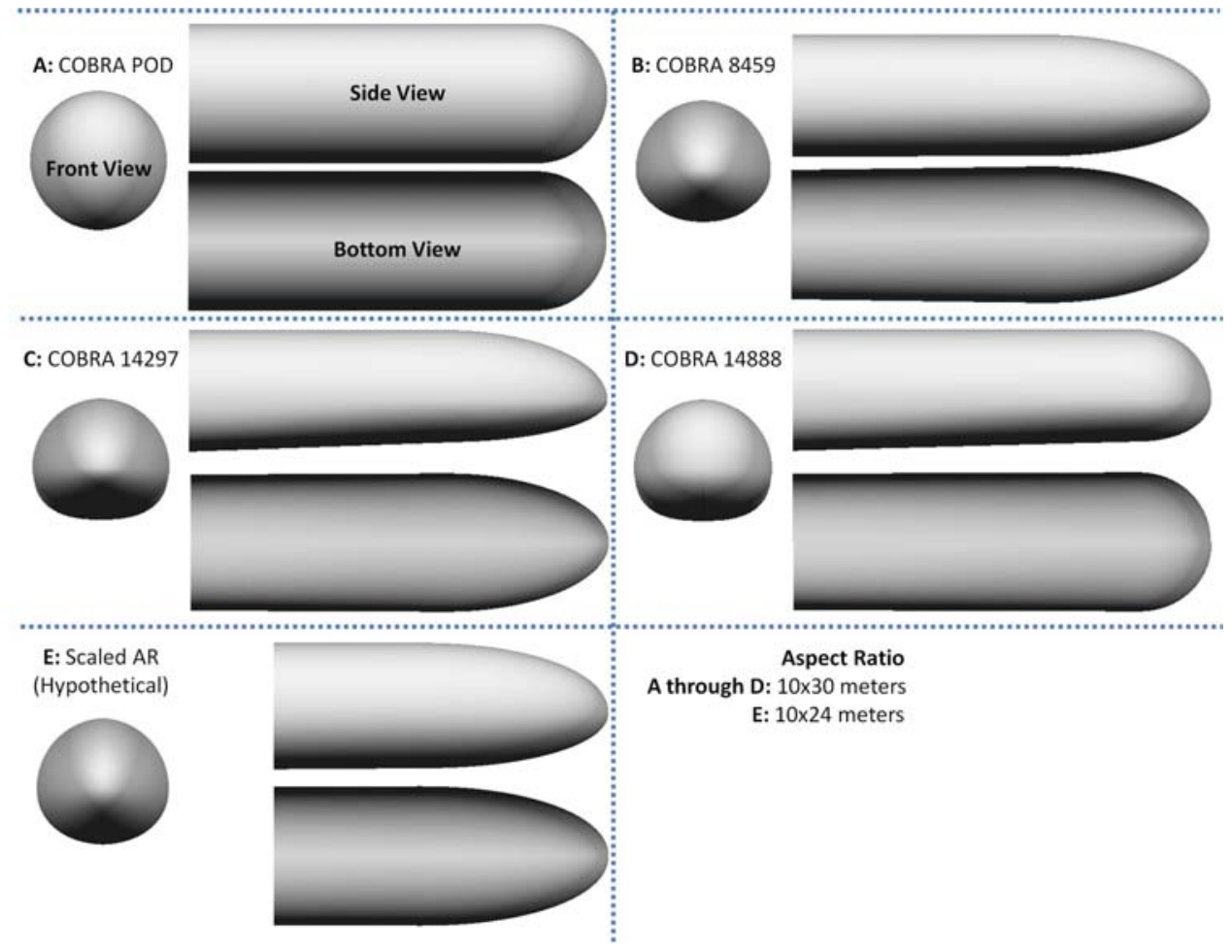

Figure 3. Dispersed geometries from COBRA study.

$C_{D}$ used to generate the baseline trajectory. The first dispersed geometry, COBRA POD, has a ballistic coefficient of $492.1 \mathrm{~kg} / \mathrm{m}^{2}$ and a wetted area of 1021 meters squared. The dispersion on the drag and lift coefficients is $-7.5 \%$ and $-13.3 \%$, respectively, from the baseline case. The next dispersed geometry is COBRA 8459 , which has the lowest $C_{D} A$ and lowest heat rate value on the pareto front. The ballistic coefficient is $406.04 \mathrm{~kg} / \mathrm{m}^{2}$ and the wetted area is 877 meters squared. The dispersion on the drag and lift coefficients is $+4.2 \%$ and $-1.4 \%$ respectively. The third dispersed geometry is COBRA 14297 , which has a $C_{D} A$ and heat rate that is mid-range on the pareto front. The ballistic coefficient is $365.7 \mathrm{~kg} / \mathrm{m}^{2}$ and the wetted area is 882 meters squared. The dispersion on the drag and lift coefficients is $+15.8 \%$ and $+9 \%$ respectively. The fourth dispersed geometry is COBRA 14888, which has the highest $C_{D} A$ and highest heat rate on the pareto 
front. The ballistic coefficient is $368.03 \mathrm{~kg} / \mathrm{m}^{2}$ and the wetted area is 937 meters squared. The dispersion on the drag and lift coefficients is $+17.7 \%$ and $+11.15 \%$ respectively. In the COBRA study it was found that COBRA 14297 had the optimal heating and weight characteristics. To test the ADG performance for an extreme case, the length of this vehicle was shortened and the aerodynamics were scaled to reflect the change in shape. This case is referred to as Scaled Aspect Ratio (AR) throughout this paper and defines the fifth dispersed geometry for this study. The ballistic coefficient is $922.4 \mathrm{~kg} / \mathrm{m}^{2}$ and the wetted area is 364.21 meters squared. The dispersion on the drag and lift coefficients is $-70 \%$ and $-85 \%$ respectively. All geometries can be found in Figure 3.

\section{Results}

Key questions must be addressed in analyzing the results of this study.

- Did the vehicle meet the separation box defined by Mach Number and Dynamic Pressure?

From the perspective of entry vehicle analysis, it is important that the vehicle reach the separation box. The box is defined by the Mach number and dynamic pressure limits found in Table 1. If the control profile does not take the vehicle within the separation box then the control profile is invalid. In Table 2 the separation condition for each vehicle from the COBRA study and the ADG are compared. The COBRA 8459, 14297, and 14888 are sufficiently guided into the separation box by the ADG. The ADG is able to guided the COBRA POD close to the separation box, where the dynamic pressure is approximately $28 \mathrm{~N} / \mathrm{m}^{2}$ larger than the upper limit. The ADG was not able to guide the Scaled AR into the separation box as the dispersions on the lift and drag coefficients were too large.

\begin{tabular}{|c|c|c|c|c|c|c|}
\hline & \multicolumn{2}{|c|}{ Separation } & $h[\mathrm{~km}]$ & $q\left[N / m^{2}\right]$ & Mach\# & \\
\hline & Nom & & 6.41251 & 1232.572 & 2.67 & \\
\hline & \multicolumn{3}{|c|}{ COBRA Study } & \multicolumn{3}{|c|}{ Guided Study } \\
\hline & $h[\mathrm{~km}]$ & $q\left[N / m^{2}\right]$ & Mach\# & $h[\mathrm{~km}]$ & $q\left[N / m^{2}\right]$ & Mach\# \\
\hline$P O D$ & 5.8525 & 1432.69 & 2.81 & 6.41471 & 1626.142 & 3.06 \\
\hline 8459 & 6.2630 & 1259.06 & 2.67 & 6.41810 & 1320.308 & 2.76 \\
\hline 14297 & 6.1497 & 1272.87 & 2.67 & 6.41139 & 1250.032 & 2.69 \\
\hline 14888 & 6.0297 & 1287.27 & 2.67 & 6.41139 & 1250.908 & 2.70 \\
\hline Scaled $A R$ & 5.9920 & 1269.39 & 2.65 & 6.41813 & 28665.294 & 12.85 \\
\hline
\end{tabular}

Table 2. Separation condition comparison between the COBRA study and Guided results.

As with any trajectory optimization, it is possible to always find a control profile that takes the vehicle to a given separation condition, which is the case for the COBRA study trajectories. In Table 2 all the COBRA trajectories are shown to meet the separation box. However, there are no constraints that identify whether any control limits were violated or if the vehicle is within a reasonable radius of the target. The worst case scenario is that the optimized trajectory is not a flight feasible trajectory and thus does not model the heating environments accurately. 

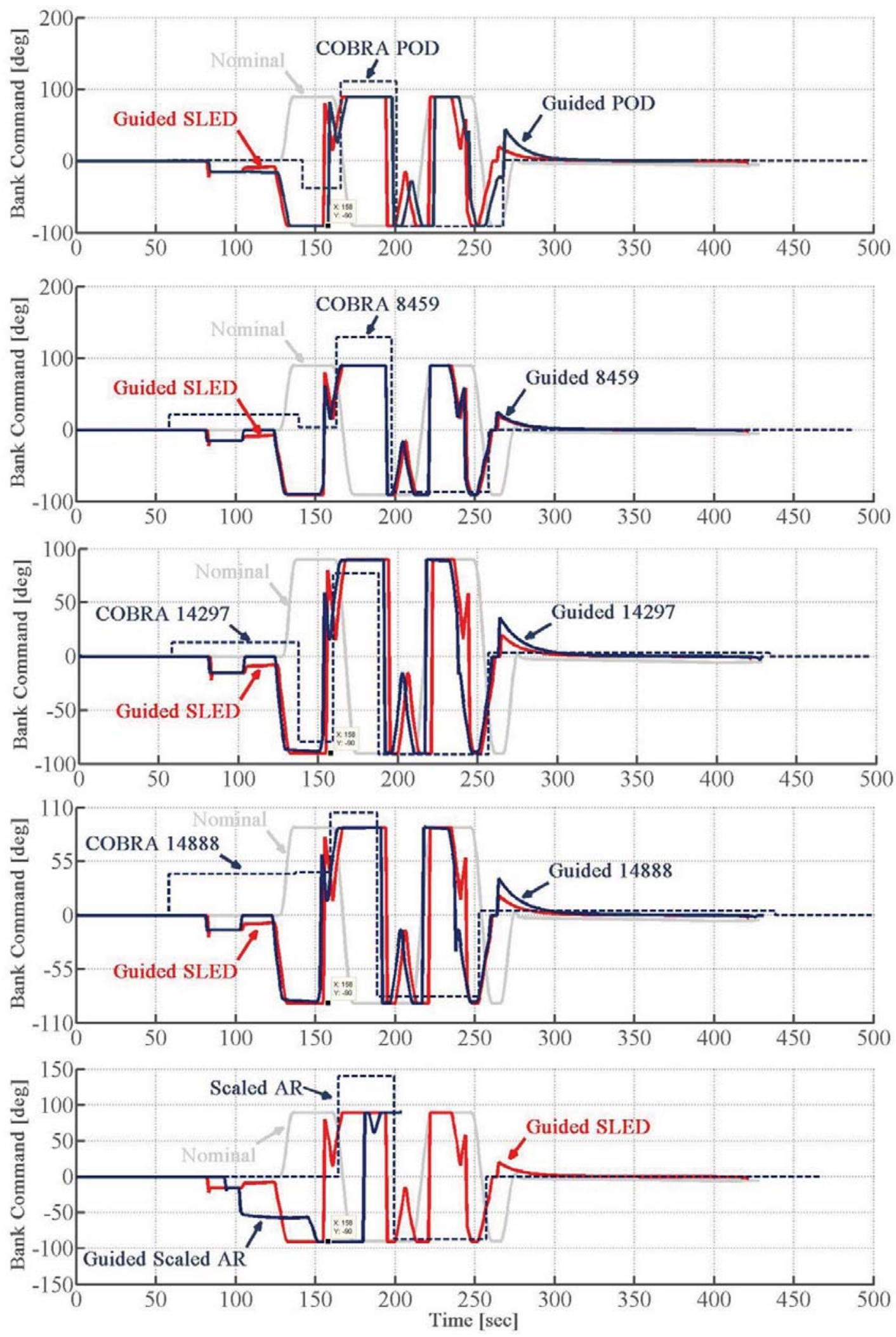

Figure 4. Bank profile comparison between the COBRA study and the ADG. 
In Figure 4, the bank profile for each dispersed geometry from the COBRA study and the ADG are plotted with respect to the nominal trajectory. It is noted in Mendeck and Craig ${ }^{15}$ that the ADG includes a limiter on the commanded vertical L/D such that bank cannot exceed $+/$ - 90 degrees. The purpose of this limiter is to ensure that the g-loading does not become excessive and violate loading constraints. This condition is especially critical for Mars entry because a full lift-up orientation $\left(0^{\circ}\right)$ for the duration of entry will guide the vehicle to a separation state well beyond the target and a full lift-down orientation $\left(180^{\circ}\right)$ will not allow adequate slowing of the vehicle, leading to catastrophic surface impact significantly short of the target. In Figure 4 the guided bank profiles (solid blue lines) for all dispersed trajectories are within the +/- 90 degree limits. The COBRA Study bank profiles (blue dashed lines) for the POD, 8459, and 14888 do not fly within the required bank limits. Thus, it is possible that the heating environments modelled do not reflect the actual in-flight behavior. Using the COBRA trajectory optimization process, a bank profile for the Scaled AR was found that took the Scaled AR to the separation box, but did not remain within the limits of $+/-90$ degrees. The ADG, however, flew a bank solution that maintained the control limits, but could not take the vehicle to the target. This results lead to the next question to consider.

- Does the prescribed bank profile guide the vehicle to within a reasonable distance of the target?

The goal of integrating a guidance algorithm in a shape optimization study is to model a realistic set of controls. This implies that the vehicle flies a realistic profile that takes the vehicle to its intended destination and does not exceed control limits. The target in this case is the separation condition for the descent stage, which is approximately $22.9 \mathrm{~km}$ from the target. This separation condition will become the 'zero' range for all trajectories and any deviation from this point indicates the amount of range error present from the separation condition. Figure 5 plots the 'separation' footprint for all trajectories considered. It should be noted that the cross range for the COBRA study trajectories

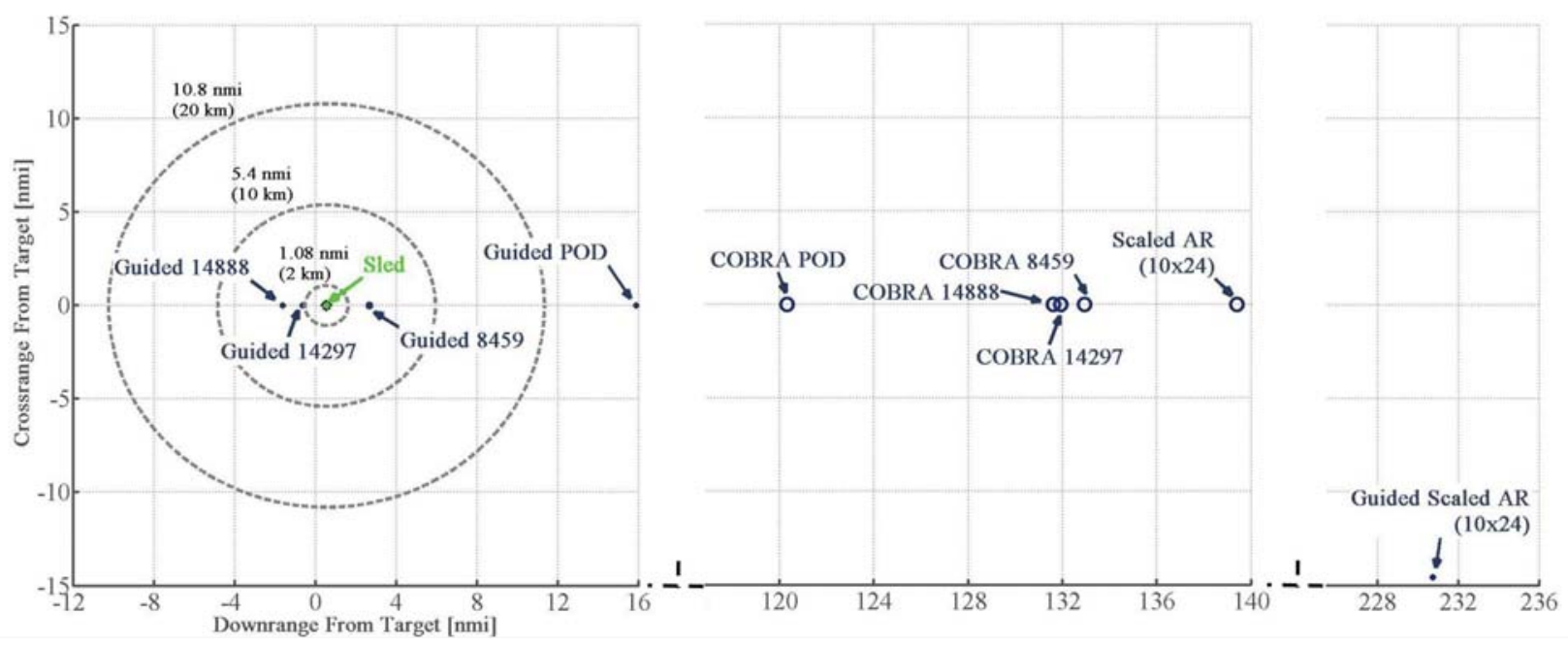

Figure 5. Separation footprint for all geometries and corresponding trajectories.

was set to zero because it was not calculated with respect to the target condition and should not be mistaken for a targeted condition. The guided trajectories show moderate performance. The COBRA 14297 dispersed geometry is guided by the ADG to within $2 \mathrm{~km}$ of the location of separation. The COBRA 8549 and 14888 vehicles were guided to within $5 \mathrm{~km}$ of the target and the COBRA POD is just outside of $30 \mathrm{~km}$ from the target. The case with the largest violation is the Scaled AR, which is far outside the target. Note that all of the trajectories from the COBRA study have considerable downrange miss distance, between $222 \mathrm{~km}$ and $257 \mathrm{~km}$. At this point, one must determine whether reaching the separation box far from the target location versus targeting both the separation box and target location dramatically changes the heating \& loading environment predictions.

- What are the differences in the aerodynamic loading and heating characteristics between each trajectory model?

At this point the peak conditions for each dispersed geometry and corresponding trajectory are com- 
pared. However, the Scaled AR is not considered from this point on as it is clearly not a valid case.

\begin{tabular}{|c|c|c|c|c|c|c|c|c|}
\hline \multicolumn{2}{|c|}{ Maximum } & \multicolumn{2}{|c|}{$q\left[N / m^{2}\right]$} & \multicolumn{2}{|c|}{ g-load $[G s]$} & \multicolumn{2}{|c|}{$\dot{q}\left[W / \mathrm{cm}^{2}\right]$} & Heatload $\left[\mathrm{J} / \mathrm{cm}^{2}\right]$ \\
\hline \multicolumn{2}{|c|}{ Nominal } & \multicolumn{2}{|c|}{11642.27} & \multicolumn{2}{|c|}{3.25} & \multicolumn{2}{|c|}{125} & 12851.97 \\
\hline & \multicolumn{8}{|c|}{ COBRA Study } \\
\hline & \multicolumn{2}{|c|}{$q\left[N / m^{2}\right]$} & \multicolumn{2}{|c|}{ g-load $[G s]$} & \multicolumn{2}{|c|}{$\dot{q}\left[W / \mathrm{cm}^{2}\right]$} & \multicolumn{2}{|c|}{ Max. Heatload $\left[\mathrm{J} / \mathrm{cm}^{2}\right]$} \\
\hline$P O D$ & \multicolumn{2}{|c|}{10896} & \multicolumn{2}{|c|}{2.59} & \multicolumn{2}{|c|}{89.495} & \multicolumn{2}{|r|}{9445.8} \\
\hline 8459 & \multicolumn{2}{|c|}{10619} & \multicolumn{2}{|c|}{2.89} & \multicolumn{2}{|c|}{84.727} & \multicolumn{2}{|r|}{11496.4} \\
\hline 14297 & \multicolumn{2}{|c|}{9555.8} & \multicolumn{2}{|c|}{2.92} & \multicolumn{2}{|c|}{81.7354} & \multicolumn{2}{|r|}{11609.5} \\
\hline \multirow[t]{3}{*}{14888} & \multicolumn{2}{|c|}{9456.1} & \multicolumn{2}{|c|}{2.92} & \multicolumn{2}{|c|}{82.524} & \multicolumn{2}{|r|}{11669.1} \\
\hline & \multicolumn{8}{|c|}{ Guided Study } \\
\hline & \multicolumn{2}{|c|}{$q\left[N / m^{2}\right]$} & \multicolumn{2}{|c|}{ g-load $[G s]$} & \multicolumn{2}{|c|}{$\dot{q}\left[\mathrm{~W} / \mathrm{cm}^{2}\right]$} & \multicolumn{2}{|c|}{ Max. Heatload $\left[\mathrm{J} / \mathrm{cm}^{2}\right]$} \\
\hline$P O D$ & \multicolumn{2}{|c|}{13347.20} & \multicolumn{2}{|c|}{3.19} & \multicolumn{2}{|c|}{97.924} & & 9175.6 \\
\hline 8459 & 111 & 7.93 & & .26 & & .986 & & 10797.8 \\
\hline 14297 & 100 & 1.16 & & .26 & & .025 & & 11114.9 \\
\hline 14888 & 100 & 6.52 & & .25 & & .382 & & 11419.1 \\
\hline
\end{tabular}

Table 3. Maximum loading and heating comparison between the COBRA study and ADG results.

In Table 3, the dynamic pressure and g-load for all four dispersed geometries, are higher for the ADG trajectories versus the COBRA trajectories. The peak heat rate for the COBRA 8459, 14297, and 14888 is larger for the COBRA study trajectories as compared to the ADG trajectories. But the COBRA POD peak heat rate for the ADG trajectory is larger than the COBRA study trajectory. The COBRA POD does not follow the same heat rate trend because this geometry and trajectory were calibrated and optimized as the new baseline for the COBRA vehicle analysis, where the basis for the new baseline is the Langley trajectory.

The overall trends that are observed can be attributed to certain aspects of the COBRA trajectory optimization process. First, the COBRA trajectories were optimized with an active dynamic pressure and g-load constraint. This contributed to control solutions that flew trajectories with excess range and excess time in-flight. In Figure 6 the extended time of the COBRA trajectories are 75-100 seconds longer than the nominal trajectory. Since the ADG trajectories must reach the same separation box and target a specific location, the in-flight time is reduced but the peak dynamic pressure \& peak g-load are slightly larger. In addition, the entry interface conditions were allowed to moderately change for the COBRA trajectory optimization. Specifically, the entry flight path angle (FPA) was made slightly steeper by approximately 1.1 degree and the entry altitude was raised by $4 \mathrm{~km}$. Since entry trajectories are very sensitive to changes in FPA, the over all result was an increase in the peak heat rate for the COBRA trajectories. The final variable for comparison is the integrated heatload, which is a primary driver to the size and mass of the thermal protection system (TPS). Heatload is the total heat rate integrated over time and is directly proportional to the thickness of the TPS. In Table 3, the heat load is shown to be larger for the COBRA trajectories versus the ADG trajectories. This is due to the extended trajectory times and higher peak heat rates for the COBRA trajectories. To illustrate the effective change in mass from lower predicted heatloads, consider a TPS for this vehicle that is an ablator. In Laub et al. ${ }^{16}$ a relationship between the TPS mass fraction and the integrated heatload was extrapolated from past entry missions that utilized an ablative TPS. The relation is as follows:

$$
\text { TPS MF }=0.091(Q)^{0.51575}
$$

where MF is the $\%$ mass fraction and Q is the total integrated heatload in $J / \mathrm{cm}^{2}$. The TPS MF for COBRA 8459, 14297, and 14888 is between 0.13 and $0.36 \%$ less for the ADG trajectories as compared 

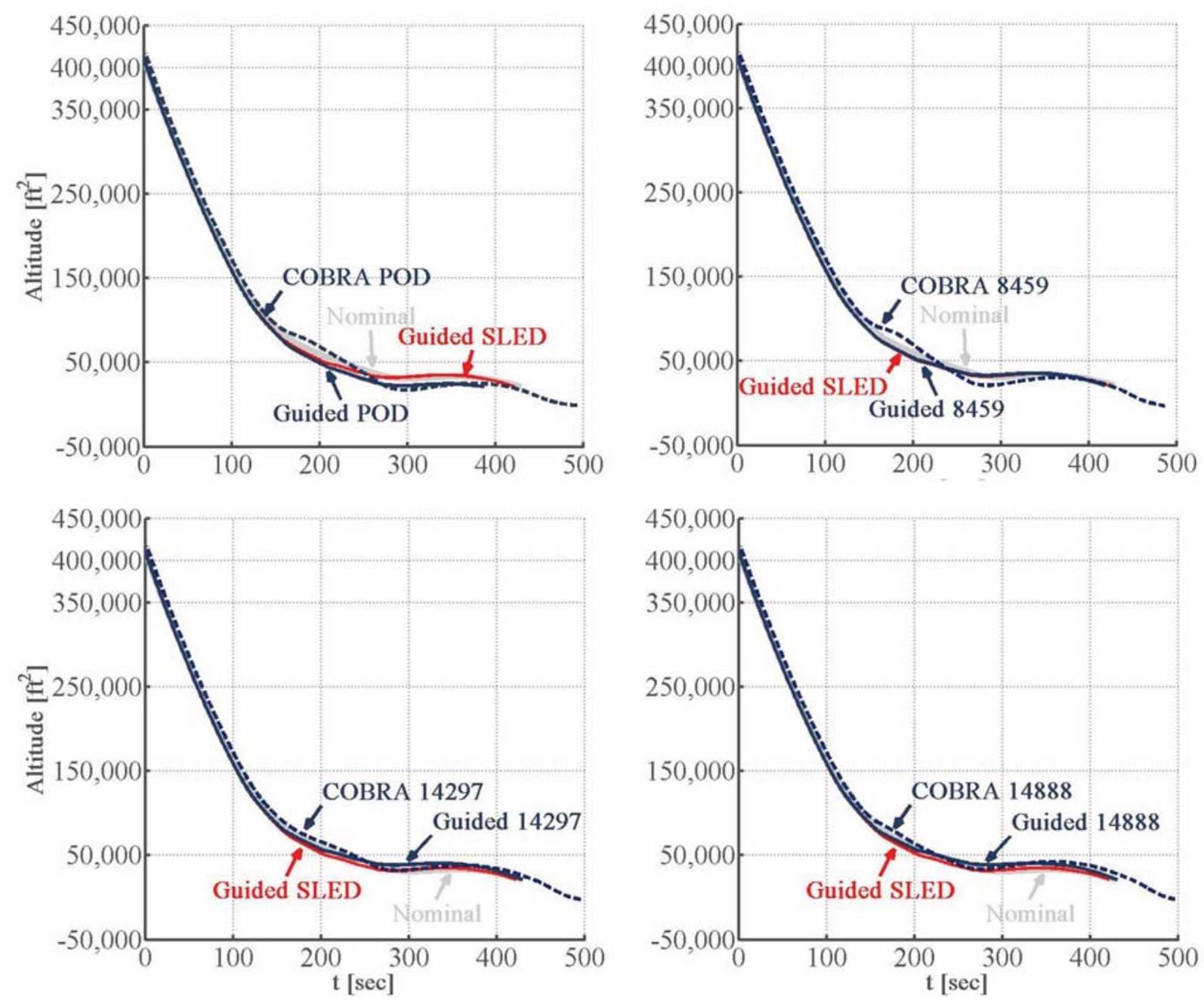

Figure 6. Comparison of altitude profile for COBRA, ADG, and the nominal case.

to the COBRA trajectories. Given a $40 \mathrm{mT}$ landed payload, the total entry mass to land that payload may be as high as $120 \mathrm{mT}$. Thus, the potential savings from using ADG trajectories to size the TPS is between 150 and $431 \mathrm{~kg}$.

\section{Conclusion and Future Work}

The results revealed that including flight feasible trajectories for a given dispersed geometry has the potential to save mass up to $430 \mathrm{~kg}$. In addition, this study also revealed that the generation of flight feasible trajectories is only as good as the performance of the guidance algorithm. It is well known that the ADG is only valid for short range, low to mid-L/D entry spacecraft performing a direct entry. In addition, the ADG will perform well as long as the dispersed conditions are within the linear perturbations used to derive the gains for the guidance law. In this study, the dispersed geometries modelled aerodynamic dispersions that ranged from $+/-1 \%$ to $+/-17 \%$ and a single extreme case was modelled where the aerodynamics were approximately $80 \%$ less than the baseline geometry. This algorithm, as expected, was able to guided the vehicle into the separation box at the target location for dispersions up to $17 \%$, but failed for the $80 \%$ dispersion cases. This pre-cursor study has provided a pathway for future work that will be required to integrate guidance into the vehicle optimization process. First, a study should be completed to determine how guidance performance varies for different baseline trajectories/geometries for a given vehicle and mission. Next, all of the geometries that generated the pareto front in the COBRA analysis should be implemented 
into the ADG so a full statistical analysis is completed to define the ADG performance limitations. Finally, an investigation of the procedures required to generate reference trajectories, for each set of geometries, that are outside of aerodynamic dispersion limits for the ADG should be completed.

\section{Acknowledgements}

The authors would like to thank Chris Cerimele, Ron Sostaric, and Eduardo Llama from NASA Johnson Space Center for their collaborative efforts on the guidance portion of this work.

\section{References}

${ }^{1}$ Davis, J.L., et al., "Guidance and Control Algorithms for the Mars Entry, Descent and Landing Systems Analysis," AIAA/AAS Astrodynamics Specialists Conference, AIAA 2010-7972, Ontario, Canada, August 2010.

${ }^{2}$ Theisinger, J.E., et al., "Multiobjective Hypersonic Entry Aeroshell Shape Optimization," 12th AIAA/ISSMO Multidisciplinary Analysis and Optimization Conference, AIAA 2008-5873, Victoria, British Columbia, Canada, September 2008.

${ }^{3}$ Johnson, J.E., et al., "Aerothermodynamic Optimization of Reentry Heat Shield Shapes for a Crew Exploration Vehicle," Journal of Spacecraft and Rocket, Vol. 44, No. 4, pp. 849-859, July-August 2007.

${ }^{4}$ Kinney, D., et al., "Conceptual Design of a 'SHARP' - CTV," AIAA-2001-2887, 200.

${ }^{5}$ Garcia, J.A., et al., "Co-Optimization of Mid Lift to Drag Vehicle Concepts for Mars Atmospheric Entry," 10th AIAA/ASME Thermophysics Conference, 2010.

${ }^{6}$ Steinfeldt, B.A., et al., "High Mass Mars Entry, Descent, and Landing Architecture Assessment," AIAA 2009-6684, AIAA Space 2009 Conference \& Exposition, Pasadena, California, September 2009.

${ }^{7}$ Loomis, M.P., Allen, G.A., "Demonstration of Integrated Trajectory/Aerothermal/TPS Sizing Design Tools for Mars Smart Landers," AIAA 2002-4508, AIAA Atmospheric Flight Mechanics Conference \& Exhibit, Monterey, California, August 2002.

${ }^{8}$ Saunders, D., et al., "Crew Transfer Vehicle Trajectory Optimization," AIAA 2001-2885, 2001.

${ }^{9}$ Kinney, D., et al., "Predicted Convective and Radiative Aerothermodynamic Environments for Various Reentry Vehicles Using CBAERO," AIAA Aerospace Sciences Meeting and Exhibit, AIAA 2006-659, 44th AIAA Aerospace Science Meeting and Exhibit, Reno, Nevada, January 2006.

${ }^{10}$ Kinney, D.J., "Aerodynamics Shape Optimization of Hypersonic Vehicles," AIAA 2006-239, 44th AIAA Aerospace Science Meeting and Exhibit, Reno, Nevada, January 2006.

${ }^{11}$ Johnson, J.E., et al., "Coupled Entry Heat Shield/Trajectory Optimization for Lunar Return," AIAA Atmospheric Flight Mechanics Conference and Exhibit, AIAA 2008-6557, Honolulu, Hawaii, August 2008.

${ }^{12}$ Dwyer-Ciancolo, A.M., et al., "Entry, Descent and Landing Systems Analysis Study: Phase 1 Report," NASA/TM-2010216720 .

${ }^{13}$ Moseley, P. E., "The Apollo Entry Guidance: A Review of the Mathematical Development and Its Operational Characteristics," TRW Note No. 69-FMT-791, TRW, December 1, 1969.

${ }^{14}$ Garcia, J.A., at al., Internal Report, NASA Ames Research Center

${ }^{15}$ Mendeck, G.F., Craig, L.E., "Entry Guidance for the 2011 Mars Science Laboratory Mission," AIAA Atmospheric Flight Mechanics Conference, AIAA 2011-6639, Portland, Oregon, August 2011.

${ }^{16}$ Laub, B., Venkatapathy, E., "Thermal Protection System Technology and Facility Needs for Demanding Future Planetary Missions," International Workshop on Planetary Probe Atmospheric Entry and Descent Trajectory Analysis and Science, Lisbon, Protugal, October 2003.

${ }^{17}$ Berning, M. J., Sagis, K.D., "User's Guide for the Simulation and Optimization of Rocket Trajectories (SORT) Program Version 7," Contract NAS 9-17900, October, 1992. 